\title{
Fragilidade em idosos na Atenção Primária à Saúde: uma abordagem a partir do geoprocessamento
}

\author{
Older adults frailty in Primary Health Care: \\ a geoprocessing-based approach
}

Fabiana Ferraz Queiroga Freitas (https://orcid.org/0000-0001-7374-1588) ${ }^{1}$

Alexsandra Bezerra Rocha (https://orcid.org/0000-0002-4814-0999) ${ }^{1}$

Ana Clara Mourão Moura (https://orcid.org/0000-0001-6823-1938) ${ }^{2}$

Sônia Maria Soares (https://orcid.org/0000-0003-3161-717X) ${ }^{2}$
${ }^{1}$ Universidade Federal de Campina Grande. R. Sergio Moureira s/n, Casas Populares. 58900000 Cajazeiras PB Brasil. fabianafqf@hotmail.com ${ }^{2}$ Universidade Federal de Minas Gerais. Belo Horizonte MG Brasil.

\begin{abstract}
This paper aimed to analyze the spatial distribution of older adults' frailty in primary health care, spatially identifying areas with a concentration of seniors, comparing the demand for care. This is an analytical study that employed spatial analysis with older adults who are frail or at risk of frailty enrolled in Primary Health Care, distributed in 32 census tracts. Concerning geolocation, we used Google Earth Pro software and "C7 GPS Data app", to elaborate the thematic and cadastral maps Qgis 2.16. In total, $43 \%$ of seniors were classified as at risk of frailty, of which 79.5\% were female, with a mean age of 75 years. The organization of the services showed an unequal distribution of the facilities in the territory, and the three health care settings present or not in some tracts and the concentration of older adults where services were difficult to access. The spatial analysis pointed out the distribution and concentration areas of frailty, favoring the comparison of social vulnerability with the possible care of health services, supporting planning actions and management of the distribution of establishments or projects to visit those in need. Thus, geoinformation tools can strengthen access to health services and provide better living conditions for seniors.

Key words Spatial Analysis, Frail Older Adult, Primary Health Care
\end{abstract}

Resumo O objetivo foi analisar a distribuição espacial da fragilidade em idosos na atenção primária à saúde identificando espacialmente áreas com concentração de idosos comparando a demanda por atendimento. Estudo analítico que utilizou análise espacial com idosos frágeis ou em risco de fragilização cadastrados na Atenção Primária à Saúde, distribuídos em 32 setores censitários. Para geolocalização utilizou-se software Google Earth Pro e "app C7 GPS Dados", para elaboração dos mapas temático e cadastral o Qgis 2.16. Classificou-se em risco de fragilização $43 \%$ dos idosos, sendo 79,5\% do sexo feminino, com média de idade 75 anos. A organização dos serviços apresentou distribuição desigual das unidades no território, com os três cenários de atenção à saúde presentes ou não em alguns setores, e concentração de idosos onde os serviços tinham difícil acesso. A análise espacial apontou a distribuição e áreas de concentração da fragilidade, favorecendo a comparação da vulnerabilidade social com a potencialidade de atendimento por parte dos serviços de saúde, dando suporte a ações de planejamento e gestão de distribuição de unidades ou projetos de visita aos necessitados. Assim, as ferramentas de geoinformação são potenciais ao fortalecimento do acesso a serviços de saúde e melhores condições de vida do idoso.

Palavras-chave Análise Espacial, Idoso Fragilizado, Atenção Primária à Saúde 


\section{Introdução}

A fragilidade é uma síndrome clínica multifatorial, influenciada por complexas e dinâmicas interações biológicas, psicológicas, cognitivas e sociais $^{1,2}$, que podem reduzir a autonomia e independência da pessoa idosa, ampliando os riscos para quedas, hospitalização, infecções, deficiências, institucionalização e morte ${ }^{3,4}$. Compreende um estado de vulnerabilidade com maiores riscos de exposições que levam a comorbidades e deficiências, em consequência, a perda de reserva do corpo humano que se relaciona a idade $e^{5,6}$.

A fragilidade pode associar-se a ocorrência de doenças crônicas não transmissíveis e dependência para o desempenho das atividades básica e instrumentais de vida diária (ABVD e AIVD), com comprometimento da cognição, humor, mobilidade e comunicação, de forma isolada ou associada, e mais acentuado entre os longevos ${ }^{7,8}$. Quando presente expõe como principais manifestações clínicas: perda de peso, exaustão, lentidão da marcha e redução da força muscular e aeróbica que afetam a funcionalidade e realização de atividades cotidianas ${ }^{3,9}$.

Atenção à temática da fragilidade vem sendo dada a nível mundial, mesmo não havendo um consenso em sua definição, a fim de compreender os fatores que determinam sua ocorrência. Trata-se da adoção de medidas preventivas que possam minimizar sua instalação e seus desfechos adversos, ampliando a expectativa de vida e reduzindo o impacto na qualidade de vida das pessoas idosas ${ }^{10}$.

No sistema de saúde brasileiro, é deficitária sua identificação, caracterizando-se um desafio para o modelo de atenção à saúde vigente, que precisa enaltecer o olhar à pessoa idosa, minimizando a progressão da fragilidade e reduzindo eventos adversos, possibilitando a manutenção da qualidade de vida com garantia da autonomia, interação social e independência, que contribuam com uma vida distante de incapacidades.

Realidade que provoca mudanças e novos desafios aos serviços de atenção à saúde, suscitando mobilização da Rede de Atenção à Saúde (RAS) na busca por melhor qualidade clínica, bons resultados sanitários e custos reduzidos por meio de atenção contínua e integral, coordenada pela Atenção Primária à Saúde (APS) ${ }^{11}$, e ofertada por equipe multiprofissional capacitada e proativa a identificar a fragilidade.

Sendo importante uma atenção interdisciplinar e multidimensional à pessoa idosa, que considere interações entre fatores físicos, psico- lógicos, ambientais e socioculturais como possíveis influenciadores do fenótipo de fragilidade. Considerando, portanto, esses aspectos são fundamentais na busca de mecanismos que ampliem a compreensão de tal fenômeno, cujos índices variam de 10 a mais de $20 \%$, entre países desenvolvidos e em desenvolvimento, como Brasil, configurando um prognóstico negativo à dependência e complicações aos anos subsequentes ${ }^{4}$.

Um dos mecanismos é dimensionar a distribuição espacial em um determinado território de diferentes fenômenos, ou seja, a espacialização e análise dessa realidade por meio do georreferenciamento das ocorrências e fenômenos espaciais, associado ao emprego de modelos de análise de distribuição espacial por geoprocessamento, existentes em Sistemas de Informações Geográficas. A chamada geografia da saúde é baseada na produção de mapas de diferentes variáveis de interesse à caracterização das vulnerabilidades e potencialidades, ofertando suporte e avaliação dos mesmos, sendo de grande importância para saúde pública e gestão em saúde, por contribuir com a identificação, localização e acompanhamento da população, controlando agravos e auxiliando no planejamento da assistência à saúde ${ }^{12,13}$.

Embora o fenótipo de fragilidade tenha sido abordado em investigações de estudos nacionais, este não foi analisado sob uma perspectiva geográfica $^{14,15}$, sendo assim, relevante a utilização do geoprocessamento com potencial para auxiliar na compreensão da distribuição espacial dos idosos e serviços de atenção à saúde, transformando o processo de avaliação, planejamento e tomada de decisões à oferta de cuidado a saúde da pessoa idosa.

Tecnologia inovadora no contexto da APS, que pode contribuir com o cuidado abrangente, comunitário e territorializado dos serviços que compõe a RAS numa perspectiva intersetorial, facilitando o gerenciamento da RAS por possibilitar maior visualização dos serviços disponíveis, melhor alocação de recursos e minimização ou dissipação de carências, sendo, portanto, importante tecnologia de apoio à saúde pública e de gestão.

Assim, justifica-se o desenvolvimento de estudos que incorporam novas contribuições a respeito dos determinantes espaciais da fragilidade com possibilidades de instrumentalizar um redirecionamento de políticas públicas que visem o cuidar das pessoas idosas, frágeis e em risco de fragilização, com vistas à garantia e manutenção da sua funcionalidade. 
Logo, para compreender a fragilidade e suas demandas de cuidado, é necessária uma avaliação multidimensional, com vistas a melhoria e manutenção da qualidade de vida, que contribua com o reconhecimento das demandas biopsicossociais, valores, crenças, sentimentos, fatores sociodemográficos, funcionais e cognitivos das pessoas idosas ${ }^{16}$.

Para isto, o Índice de Vulnerabilidade Clínico Funcional-20 (IVCF-20) trata-se de um instrumento desenvolvido no Brasil, a partir de outros instrumentos sobre a triagem da fragilidade já citados na literatura, viável ao rastreio da fragilidade na APS, pela possibilidade em indicar pontos necessários à intervenções capazes de melhorar a autonomia e independência do idoso, prevenindo o declínio funcional, institucionalização e óbito; também apropriado para utilização em clínicas geriátricas, centros de convivência, serviços de urgência e emergência e instituições de longa permanência ${ }^{17}$.

O objetivo deste estudo foi analisar a distribuição espacial da fragilidade em idosos na atenção primária à saúde identificando espacialmente as áreas com concentração (média e alta prioridade de idosos) e comparando a demanda por atendimento existente com a distribuição da rede de serviços de saúde instalada.

\section{Métodos}

Estudo analítico, que utilizou análise espacial de idosos frágeis e em risco de fragilização, registros cadastrados nas 12 Unidades Básicas de Saúde (UBS) e serviços de atenção a saúde da região geográfica imediata do município de Pombal, Paraíba, Brasil, que abrange uma área de 892,98 $\mathrm{km}^{2}$, população aproximada de 32.110 habitantes e densidade demográfica de 36,17 (habitantes $\left./ \mathrm{km}^{2}\right)^{18}$, desenvolvido no período de janeiro a março de 2017.

A população do estudo foi composta pelos idosos com 65 anos ou mais cadastrados nas UBS, sendo a amostra 183 idosos classificados frágeis ou em risco de fragilização, após rastreio da fragilidade, a partir do IVCF-20 proposto por Moraes et al. ${ }^{17}$ Instrumento constituído por 20 questões, distribuídas em oito dimensões: idade; autopercepção da saúde; atividades de vida diária (três instrumentais e uma básica); cognição; humor/comportamento; mobilidade (alcance, preensão e pinça; capacidade aeróbica/muscular; marcha e continência esfincteriana); comunicação (visão e audição); e presença de comorbi- dades múltiplas (polipatologia, polifarmácia e/ ou internação recente). $\mathrm{O}$ instrumento apresenta pontuação total de 40 pontos, classificando o idoso robusto quando escore de 0 a 6 pontos, em risco de fragilização de 7 a 14 pontos, e frágil $\geq$ 15 pontos.

Para rastreio da fragilidade os critérios de inclusão foram: idosos com idade igual ou superior a 65 anos, cadastrados na UBS há, pelo menos, seis meses, sem déficit locomotor que impossibilitasse a participação no estudo, considerando apenas um idoso por residência. Foram excluídos idosos ausentes de suas residências, após três tentativas de visita, hospitalizados, acamados e com diagnóstico médico confirmado de demência, por inviabilizar a participação no estudo frente ao declínio de memória que interfere no desempenho cognitivo do idoso.

Após rastreio da fragilidade, as coordenadas com dados dos idosos e serviços de assistência à saúde foram pontuadas manualmente em áreas de difícil acesso no programa Google Earth $\mathrm{Pro}^{\circledR}$ (GOOGLE, 2017) ou in loco pelo aplicativo C7 GPS Dados, que captura coordenadas de posicionamento de pontos de interesse, com informações expressas em coordenadas geográfica, hexadecimais e Universal Transversa de Mercator, salvas em arquivo KML e convertendo no programa QGis 2.16 para shape, no sistema de projeção SIRGAS 2000 - UTM (Universal Transversa de Mercator). Foram necessários ambos os processos (em escritório e in loco) tendo em vista algumas limitações de localização da informação nas imagens de satélite, por dificuldade de identificação de domicílios a serem registrados em vista de topo e em baixa resolução.

Para desenvolvimento da análise espacial foram utilizadas informações sobre o número de habitantes por setor censitário ${ }^{18}$, unidade geográfica utilizada pelo Instituto Brasileiro de Geografia e Estatística (IBGE) que corresponde ao controle cadastral de coleta para o censo demográfico. O núcleo urbano do município de Pombal é constituído por 32 setores censitários, subdivididos em cinco bairros. Os dados da malha dos setores censitários, recortes para fins estatísticos, base de faces de logradouros, estado e municípios utilizados neste artigo podem ser obtidos no site do IBGE em Geociências Downloads, sendo eles os dados oficiais brasileiros sobre variáveis socioeconômicas (faixas etárias, escolaridade, renda, infraestrutura existente, entre outros), agrupados pela unidade territorial de setor censitário, utilizados como controle espacial de coleta para o censo demográfico ${ }^{18}$. Os 
setores censitários constituem-se em áreas contíguas diretamente relacionada à divisão político administrativa, recortados em eixos de vias e por tipologias de uso do solo em porções de bairros, além de separados por setores urbanos e rurais.

O sistema SIRGAS/2000 foi utilizado para elaboração dos mapas no software QGis 2.16, em função de se o sistema oficial da cartografia brasileira hoje. Os polígonos de setores censitários foram associados às tabelas alfanuméricas contendo dados das variáveis socioeconômicas através de processo de "join", pois as entradas nas tabelas são individualizadas pelo código do setor censitário e isto favorece a indexação e as consultas espaciais. Realizada a associação foram realizadas consultas temáticas sobre a espacialização de informações sobre os idosos frágeis e em risco de fragilização. Foram elaborados 7 mapas temáticos a partir dos dados coletados em campo e comparados com os dados socioeconômicos do IBGE: porcentagem pelo número total de habitantes e número total de idosos com 65 anos ou mais; percentual de idosos com idade igual ou superior a 65 anos percentual de idosos frágeis; percentual de idosos frágeis por idade; percentual de idosos em risco de fragilização, percentual de idosos em risco de fragilização por idade; percentual de idosos frágeis e em risco de fragilização total e por idade; e distribuição das áreas com média e alta prioridade.

O estudo foi aprovado pelo Comitê de Ética em Pesquisa da Universidade Federal de Minas Gerais, obedecendo a todos os trâmites legais, e, respeitando a Resolução 466/12 ${ }^{19}$ do Conselho Nacional de Saúde. Os participantes foram informados sobre os objetivos do estudo e assinaram o Termo de Consentimento Livre e Esclarecido, garantindo-se sigilo dos dados e anonimato.

\section{Resultados}

Neste trabalho o universo mapeado representou a distribuição geoespacial onde estão localizados o contingente da população idosa, considerando especialmente, os idosos frágeis e aqueles em risco de fragilização. Houve predomínio de idosos em risco de fragilização (132, 43,0\%), do sexo feminino $(105,79,5 \%)$ e média de idade de 75,51 anos. Foram classificados frágeis 51 idosos $(16,6 \%)$ e robustos $(124,40,4 \%)$.

A organização dos serviços que compõem a RAS apresentou distribuição desigual das UBS no território. Observa-se neste sentido, que essa distribuição se encontra descentralizada das áreas de abrangência das equipes de saúde da família. Foram evidentes setores censitários, onde encontrou-se distribuídos os três cenários de atenção à saúde, e, setores sem serviços de atenção à saúde, necessitando restruturação desses serviços na perspectiva de ser possível o cumprimento dos princípios e diretrizes do Sistema Único de Saúde pela secretaria municipal de saúde e poder público (Figura 1).

A alta concentração dos idosos com 65 anos ou mais predominou em seis setores censitários (Figura 1). Nessas áreas de alta concentração há apenas um serviço de saúde de atenção secundária e dois de atenção primária, para o atendimento de 4.430 pessoas idosas, com evidências de 12 idosos frágeis e $23 \mathrm{em}$ risco de fragilização.

As Figuras 2A/B e 2C/D representam um conjunto de mapas no recorte do núcleo urbano que traz o percentual de idosos frágeis e em risco de fragilização. A Figura 2A mostra dois setores com alto percentual de idosos frágeis e três serviços de atenção à saúde. A Figura $2 \mathrm{~B}$ exibe áreas com baixo percentual de idosos com idade de 65 anos ou mais.

$\mathrm{Na}$ Figura 2C, quando é analisada a distribuição dos idosos em risco de fragilização observase a existência de dois setores com alto percentual desses idosos e apenas um serviço de atenção à saúde para um quantitativo de 102 idosos, onde 09 são considerados em risco de fragilização.

Após análise para se obter a proporção por idade, percebe-se na Figura 2D um setor sem serviço de atenção à saúde que necessita de atenção imediata, este, com uma população de 37 idosos acima de 65 anos, sendo 7 em risco de fragilização.

A Figura 3 mostra que os idosos se distribuem geograficamente por grande parte da área central urbana do município, mas há destaque para três setores censitários quanto à concentração das áreas de prioridade média e alta para $\mathrm{o}$ atendimento, por serem idosos frágeis ou em risco de fragilização. Juntamente à espacialização dos idosos, são localizadas as unidades de atendimento, serviços primário, secundário e terciário. A análise da localização dos atendimentos em comparação com as porções da cidade mais necessitadas alerta para a necessidade de revisão de recursos, pois nas proximidades de setor de média prioridade há três unidades de atendimento, nos três níveis. Contudo, nas áreas de maior prioridade para o atendimento, em um a delas há apenas uma unidade de serviços primários e em outra área não há unidades de atendimento nas redondezas. As áreas de alta prioridade são formadas por 2 setores censitários e população de 16 idosos 


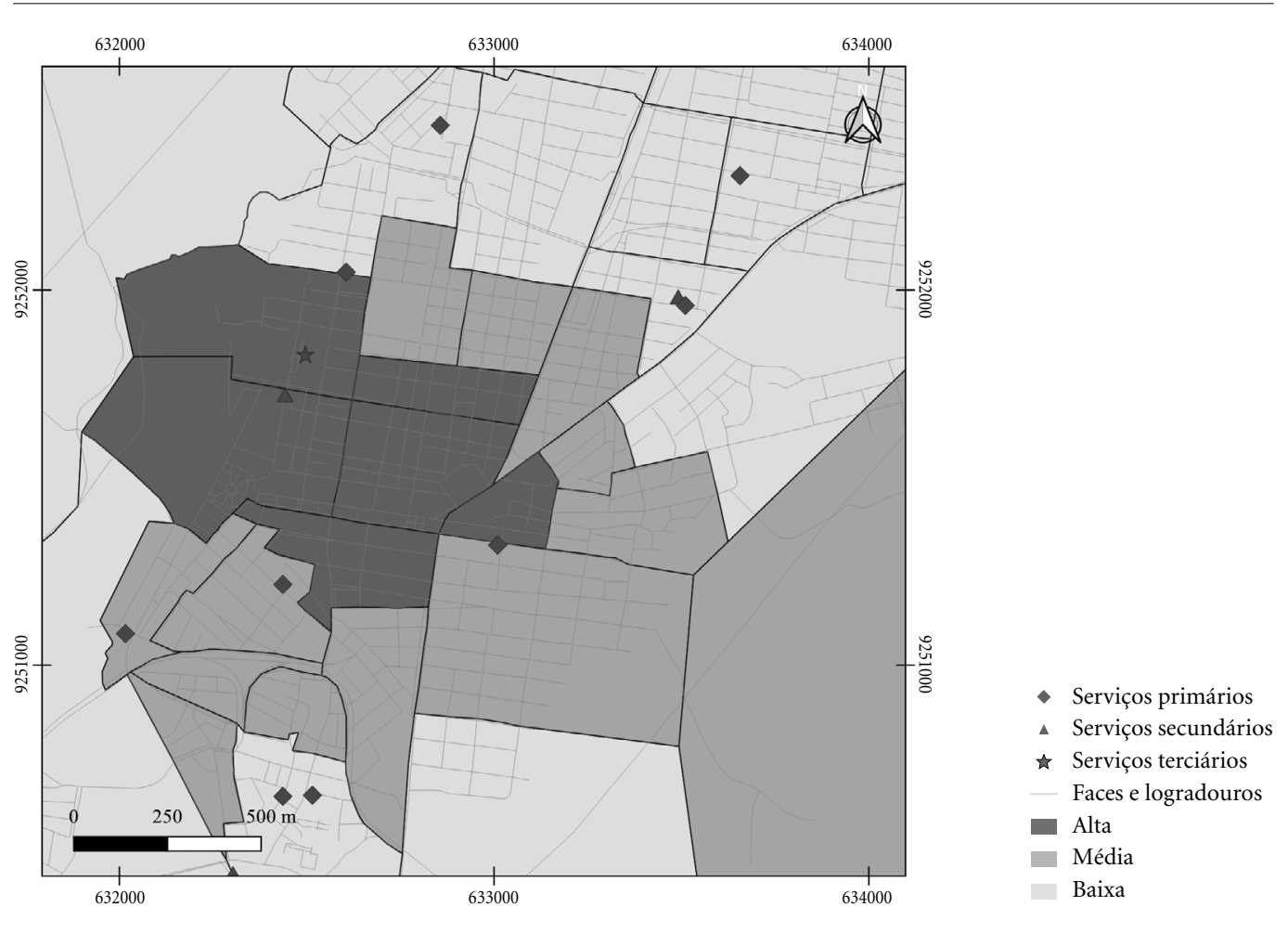

Figura 1. Distribuição das áreas com idosos em idade igual ou superior a 65 anos por setor censitário e espacialização dos serviços de atenção à saúde. Pombal-PB, 2017.

Fonte: Base Vetorial do IBGE SIRGAS 2000 UTM 24 S, Dados coletados pelos autores-2017.

frágil ou em risco de fragilização, ressaltando-se que no setor ao norte não há serviços de atenção à saúde. Como consequência, será necessário realizar gestão de recursos para compatibilizar vulnerabilidades e potenciais.

\section{Discussão}

Os mapas obtidos por meio da análise espacial permitiram um diagnóstico situacional da distribuição espacial do evento da fragilidade em idosos no município de Pombal-PB. Assim, foi possível observar que a fragilidade entre os idosos por setor censitário distribui-se de maneira aleatória, identificando os setores censitários com maior concentração de casos, dado importante à alocação de condutas e ações visando o diagnóstico precoce da fragilidade.

A caracterização do perfil da amostra demonstrou que o risco de fragilização é mais frequente em idosos do sexo feminino. Estudos conduzidos em outras cidades brasileiras tam- bém encontraram dados semelhantes. Um dos aspectos discutido na literatura, explica que isto pode resultar da fragilidade intrínseca ao sexo, pela menor concentração de massa magra e redução da força muscular, quando comparada aos homens. Além disso, outras questões são relevantes, tais como a maior vulnerabilidade a fatores extrínsecos como a sarcopenia ${ }^{20,21}$.

O fenômeno da feminização da velhice é um risco intrínseco ao desenvolvimento da fragilidade, agravado com a sobrecarga de doenças em idades avançadas ${ }^{3}$. Outro fator que pode contribuir com essa predominância é a maior longevidade em relação aos homens, que amplia o risco de incidência para doenças crônicas não-transmissíveis, inerente as modificações fisiológicas e funcionais do envelhecimento humano, podendo causar limitações que as tornam com maiores riscos de eventos adversos ${ }^{22}$.

Estudos internacionais apontam a fragilidade em idosos americanos com 65 anos ou mais, com maior concentração no sexo feminino e índices de $10 \%$ a $25 \%$, que aumentam para mais de $40 \%$ 


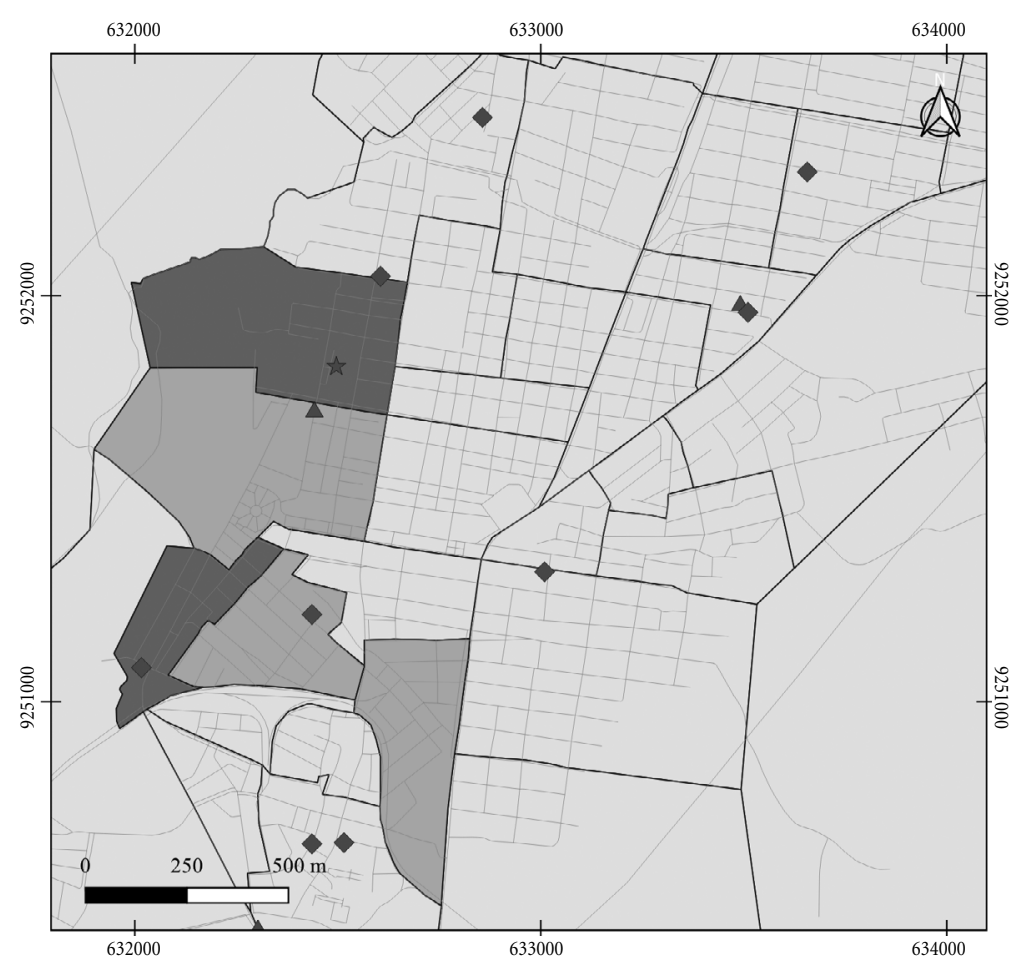

- Serviços primários

- Serviços secundários

* Serviços terciários Faces e logradouros

- Alta

- Média

- Baixa



- Serviços primários

^ Serviços secundários

* Serviços terciários

- Faces e logradouros

- Alta

- Média

- Baixa

Figura 2A/B. Distribuição das áreas com idosos frágeis por setor censitário e idade, espacialização dos serviços de atenção à saúde. Pombal-PB, 2017.

Fonte: Base Vetorial do IBGE SIRGAS 2000 UTM 24 S, Dados coletados pelos autores-2017. 




- Serviços primários

- Serviços secundários

- Serviços terciários

- Faces e logradouros

- Alta

- Média

Baixa

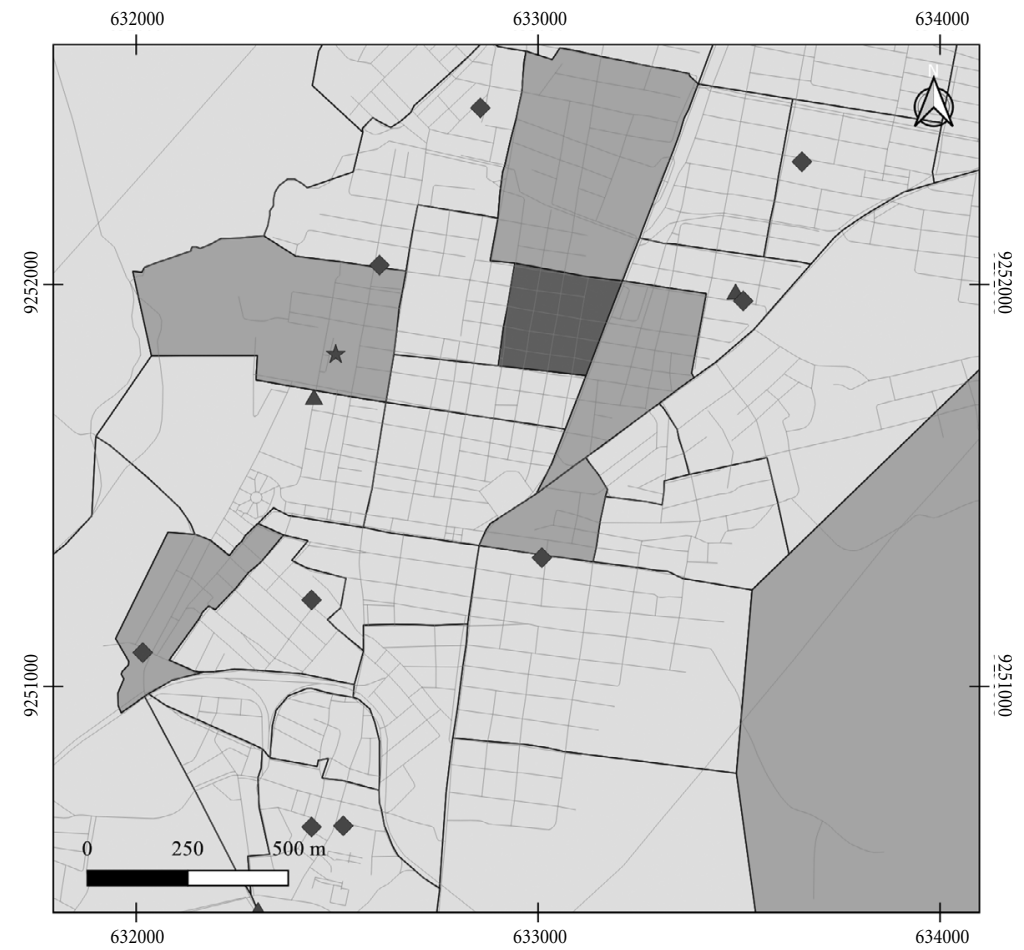

- Serviços primários

- Serviços secundários

Serviços terciários

- Faces e logradouros

- Alta

Média

- Baixa

Figura 2C/D. Distribuição das áreas com idosos em risco de fragilização por setor censitário e idade, espacialização dos serviços de atenção à saúde. Pombal-PB, 2017. 


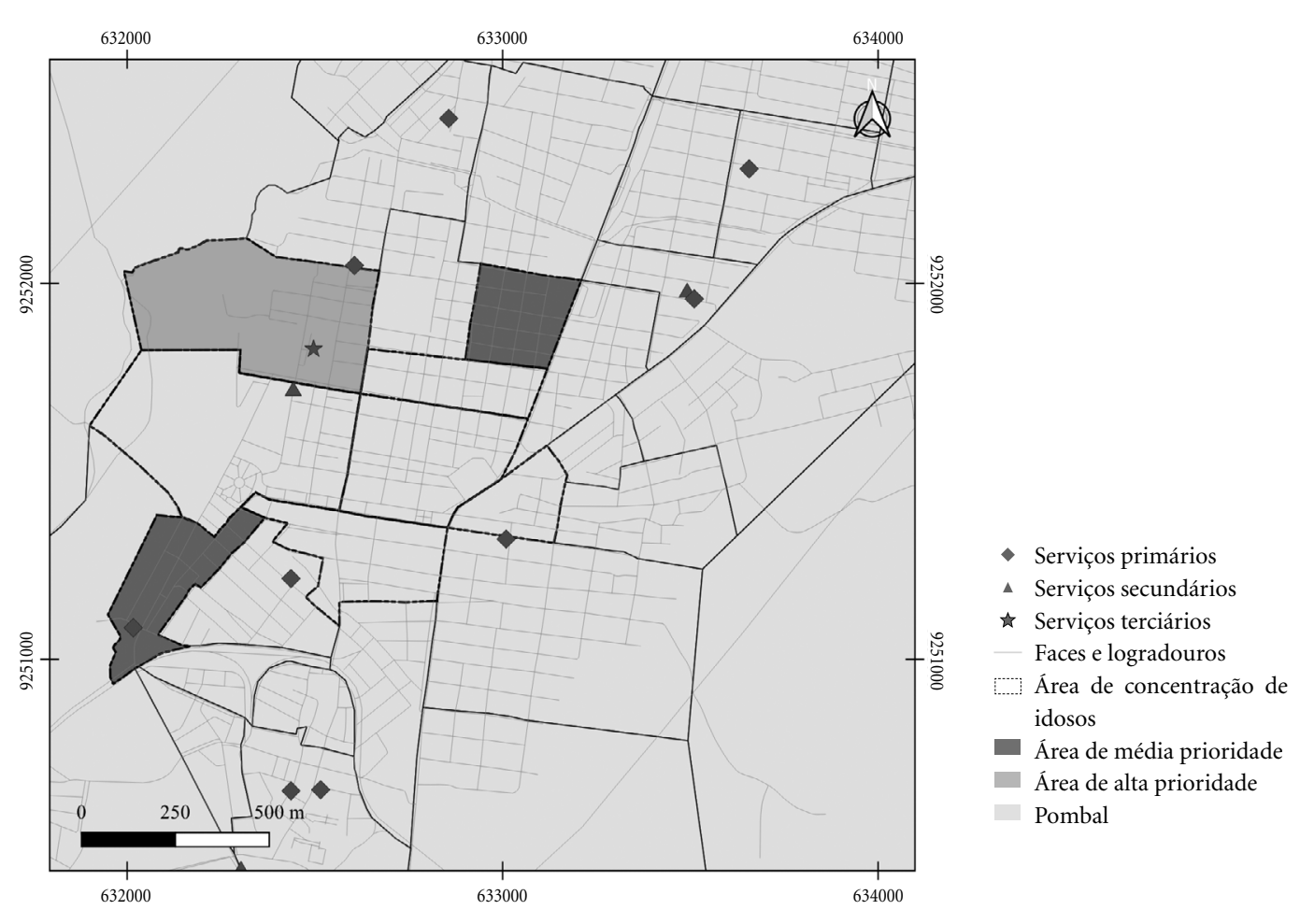

Figura 3. Distribuição das áreas com concentração, média e alta prioridade de idosos por setor censitário e espacialização dos serviços de atenção à saúde. Pombal-PB, 2017.

Fonte: Base Vetorial do IBGE SIRGAS 2000 UTM 24 S, Dados coletados pelos autores-2017.

na faixa etária superior a $80 \operatorname{anos}^{9,22}$, despertando a necessidade de um olhar atento da equipe multiprofissional.

Para atender a esse perfil, o planejamento das ações de saúde precisa garantir acessibilidade aos serviços, que consequentemente não possuem informações sobre as condições dos idosos. Tornar o serviço acessível auxilia no alcance dessas informações, estabelecendo intervenções capazes de melhorar a autonomia e independência do idoso, em um contexto de RAS, prevenindo o declínio funcional, institucionalização e óbito.

Com isso, práticas que propiciem o estilo de vida ativo e saudável, considerando as especificidades e singularidades dos idosos, tais como: alimentação saudável, atividade física, imunização, prevenção à hipertensão, tabagismo, dislipidemia e quedas, podem ser incentivadas, sob a perspectiva de dificultar eventos indesejáveis como fraturas provenientes de quedas, depressão e dependência funcional, que acarretam maiores custos ao SUS ${ }^{23}$.
A análise espacial demonstrou os setores censitários com alto percentual de idosos frágeis e em risco de fragilização, em que a vulnerabilidade clínico funcional se distribuiu heterogeneamente, definindo-os como setores prioritários de atuação. Será necessário estabelecer plano estratégico de gestão de recursos que faça a compatibilidade entre vulnerabilidades e potenciais existentes.

Visando facilitar essa demanda, o IVCF-20 caracteriza-se como instrumento de rastreio da vulnerabilidade clínico funcional, que pode ser aplicado por profissionais de nível superior e médio, treinados, indicando intervenções interdisciplinares para melhorar a autonomia e independência do idoso, planejando ações da APS com definição dos grupos de risco para atendimento diferenciado na $\mathrm{UBS}^{17}$, possibilitando estabelecer um plano de cuidados individualizado na tentativa de promover melhor qualidade de vida.

A utilização deste instrumento pode fornecer dados que se bem utilizados contribuem com melhores condições de vida. Importante atribu- 
to para promoção da saúde, prevenção e manejo da fragilidade, que se diagnosticada previamente, torna-se mais fácil a regressão. No âmbito do SUS, que tem a APS como porta de entrada preferencial aos serviços de atenção à saúde, poderá contribuir com o cuidado integral à pessoa idosa, reconhecendo suas demandas biopsicossociais.

Os setores com maiores percentuais de fragilidade e em risco de fragilização permitiu uma visão imediata da distribuição dos idosos auxiliando na compreensão do risco das áreas coletivas ou com aglomeração de pessoas. Configuramse como setores com maior número de pessoas idosas $^{18}$, constituídos por ruas em proximidade à área hospitalar, escolar e comercial, além de uma comunidade quilombola urbana. Informação que respalda e justifica a atenção à saúde com foco na prevenção da vulnerabilidade clínico funcional, uma vez que são elevados os índices de fragilidade e risco de fragilização.

Assim, a análise espacial a partir desses índices é uma ferramenta valiosa à estratificação de risco na diversificada população idosa. Sua detecção e prevenção prévia podem postergar à incapacidade e morte prematura, quando precocemente intervenções são aplicadas, possibilitando redução dos riscos ${ }^{22}$.

A espacialização dos serviços de atenção à saúde permitiu perceber sua distribuição no município, e que, essa distribuição está distante de atender as reais necessidades dos idosos frágeis e em risco de fragilização, pois os serviços de transportes públicos para locomoção à UBS e demais serviços que compõe a RAS municipal, são restritos, caracterizando uma região com precário planejamento de infraestrutura física, áreas extensas e microáreas distantes da UBS. Fato que sugere remanejamento de famílias e/ou ruas entre UBS mais próximas, na perspectiva de garantir maior cobertura de atenção ao idoso.

A atenção ofertada a partir da RAS do município onde o estudo foi desenvolvido ocorre com limitada disponibilidade de especialistas em geriatria e gerontologia, dificuldade de acesso aos demais cenários de atenção devido a restritas ações de referência e contrarreferência, inviabilizando a continuidade e integralidade da atenção, bem como os meios disponíveis a resolutividade das especificidades da pessoa idosa.

Condição também presente na região metropolitana de São Paulo, que referiu ausência de serviços e ações de saúde específicas ao atendimento da população idosa, ou incipientes iniciativas para o atendimento integral desse segmento, com ausentes linhas de cuidado ou fluxo de referência e contrarreferência. Evidenciando serviços deficientes e sem integração, com falta de facilitação de acesso aos diversos cenários de complexidade da atenção, e poucos investimentos na qualificação da equipe multiprofissional para atenção da pessoa idosa ${ }^{24}$.

Fato que reflete na continuidade do cuidado, coordenada pela APS, que precisa identificar as especificidades da população adscrita, promovendo a articulação com toda RAS, para sanar as necessidades, sejam elas geográficas, organizacionais, culturais, financeiras ou de acesso ${ }^{25}$, para assim, ampliar e qualificar o acesso humanizado e integral, fortalecendo o vínculo entre profissionais de saúde e idosos, facilitando a operacionalização da RAS.

Cenário, que apesar da boa taxa de cobertura da $\operatorname{APS}(100 \%)^{26}$, demanda ações para atenção à saúde do idoso pautada na coletividade e integrada às especificidades da pessoa idosa, que incentivem sua proatividade, com atenção especializada e articulada a RAS. Para desta forma, ser possível o alcance da equidade, integralidade e universalidade.

Sendo desafiador garantir aos idosos integralidade e acesso aos serviços de atenção à saúde, realidade excludente e desigual a toda população brasileira $^{27}$. Logo, compreender as áreas de média e alta prioridade fornece possibilidades de planejamento da assistência e contribui com a tomada de decisão acerca da ideal localização dos serviços de atenção á saúde, mais adequado quando pautado em características locais, sejam elas geográficas, socioeconômicas, culturais e/ou políticas.

Garantir acessibilidade envolve investimento de gestão na infraestrutura, que pode dispensar tempo prolongado e elevados recursos financeiros, com aumento da cobertura dos serviços de atenção à saúde, melhoria da rede de água, esgoto, moradias e educação, além da criação de áreas de convivência e lazer com oferta de transporte e vias públicas seguras e com qualidade ${ }^{28}$.

Outra dificuldade apresentada pelo município, refere-se às barreiras arquitetônicas quanto à acessibilidade nas vias, pois as vias sem manutenção ou sem condições adequadas para o deslocamento de pedestres com limitações de locomoção podem representar potenciais riscos para quedas dos idosos, favorecendo um deslocamento mais lento e prolongado, com excessiva exposição solar.

Nessa conjuntura, a ausência ou rotatividade de profissionais nas UBS e os problemas de infraestrutura, representam ruptura a atenção à saúde do idoso frágil ou em risco de fragilização, que 
pode apresentar perda da autonomia e independência, tornando-se mais vulneráveis a traumas, infecções ou alterações psicológicas, que traduz maior necessidade de assistência à saúde.

Diante desse quadro, os idosos frágeis e em rico de fragilização necessitam de UBS com arquitetura e entornos que garantam o acesso seguro e permitam boa mobilidade, com vistas à reabilitação de eventos que comprometem suas atividades básicas e instrumentais de vida diária. Sendo papel da gestão municipal, garantir o acesso e a continuidade da atenção à saúde, independente do nível de autonomia da pessoa idosa ${ }^{29}$.

Desta forma, para auxiliar as ações que resultem na melhoria da acessibilidade, a análise espacial permitiu identificar regiões não atendidas, possibilitando intervenções para o fortalecimento da RAS de forma a efetivar a referência e contrarreferência, na perspectiva de os serviços atuarem na modificação da frequência, distribuição dos agravos e desigualdades intersetoriais e no cumprimento da universalidade e integralidade previstas no SUS, que resultarão no envelhecimento saudável e promoção da qualidade de vida $^{30}$.

Logo, a análise espacial é uma ferramenta que auxilia no controle e prevenção dos problemas de saúde, suscitando mudança no estilo de vida e adoção de práticas saudáveis que prorroguem a independência, autonomia e expectativa de vida, com destaque a ampliação do acesso aos serviços de atenção à saúde ${ }^{20}$.

Em função disso, práticas e intervenções descentralizadas que considerem a capacidade e individualidade de cada idoso podem ser desenvolvidas, dentre as quais, citam-se grupos de convivência para a população idosa, como estratégia que colabora e promove o envelhecimento ativo e autônomo com inclusão e participação social.

\section{Conclusão}

A partir da análise espacial, foi possível conhecer a distribuição da fragilidade entre os idosos residentes no município de Pombal-PB e a clara visualização das áreas de maior concentração de idosos frágeis e em risco de fragilização, que consequentemente precisam de maior esforço público e da equipe multiprofissional para o planejamento, monitoramento e avaliação de intervenções capazes de possibilitar a autonomia e independência da pessoa idosa. Além de ações que contribuam com a cobertura da APS, melhorando o acesso aos serviços de atenção à saúde na perspectiva de RAS resolutiva, e que contemple as especificidades do idoso.

Caracterizando-se como importante ferramenta para o fortalecimento de ações de combate a fragilidade e de acesso aos serviços de atenção à saúde, assessorando na tomada de decisão e na implantação de políticas públicas que contribuam com melhores condições de vida e saúde da população idosa.

O estudo mostrou algumas limitações no levantamento de discussões por se tratar de análise espacial com idosos, havendo poucas informações provenientes de outros estudos ou bibliografia publicada. No que diz respeito a processos de análise espacial foram estudados temas de geografia da saúde, mas publicações como a presente abrem portas para as discussões específicas das variáveis relacionadas à saúde do idoso. 


\section{Colaboradores}

FFQ Freitas participou da concepção e delineamento, revisão da literatura, coleta dos dados, contribuiu na análise e interpretação dos dados, redação da versão inicial e final do manuscrito. AB Rocha participou da coorientação do projeto, contribuiu na análise espacial e interpretação dos dados, contribuiu na análise e revisão crítica do manuscrito. ACM Moura participou da coorientação do projeto, contribuiu na análise espacial e interpretação dos dados, contribuiu na análise e revisão crítica do manuscrito. SM Soares responsável pela orientação do projeto, análise e interpretação dos dados, revisão crítica e aprovação da versão final do manuscrito.

\section{Referências}

1. Rockwood K, Bergman H. Frailty: a report from the 3rd Joint Worshop of IAGG/WHO/SFGG, Athens, January 2012. Can Geriatr J 2012; 15(2):31-36.

2. Morley, JE, Vellas B, Abellan VKG, Anker SD, Bauer JM, Bernabei R, Cesari M, Chumlea WC, Doehner W, Evans J, Fried LP, Guralnik JM, Katz PR, Malmstrom TK, Mccarter RJ, Robledo LMG, Rockwood K, Haehling SV, Vandewoude MF, Walston J. Frailty consensus: a call to action. J Am Med Dir Assoc 2013; 14(6):392-397.

3. Fried LP, Tangen CM, Walston J, Newman AB, Hirsch C, Gottdiener J, Seeman T, Tracy R, Kop WJ, Burke G, McBurnie MA. Frailty in older adults: evidence for a phenotype. J Gerontol A Biol Sci Med Sci 2001; 56(3):146-156.

4. Zhu Y, Liu Z, Wang Y, Wang Z, Shi J, Xie X, Jin L, Chu $X$, Wang $X$. Agreement between the frailty index and phenotype and their associations with falls and overnight hospitalizations. Arch Gerontol Geriatr 2016; 66:161-165.

5. Dent E, Lien C, Lim WS, Wong WC, Wong CH, Ng TP, Woo J, Dong B, de la Vega S, Hua Poi PJ, Kamaruzzaman SBB, Won C, Chen LK, Rockwood K, Arai H, Rodriguez-Mañas L, Cao L, Cesari M, Chan P, Leung E, Landi F, Fried LP, Morley JE, Vellas B, Flicker L. The Asia-Pacific Clinical Practice Guidelines for the Management of Frailty. J Am Med Dir Assoc 2017; 18:564575.

6. Santos PHS, Fernandes MH, Casotti CA, Coqueiro RS, Carneiro JAO. Perfil de fragilidade e fatores associados em idosos cadastrados em uma Unidade de Saúde da Família. Cien Saude Colet 2015; 20(6):1917-1924.

7. Campolina AG, Adami F, Santos JLF, Lebrão ML. A transição de saúde e as mudanças na expectativa de vida saudável da população idosa: possíveis impactos da prevenção de doenças crônicas. Cad Saude Publica 2013; 29(6):1217-1229.

8. Moraes EN. Atenção à saúde do idoso: aspectos conceituais. Brasília: OPAS; 2012.

9. Fried LP. Interventions for Human Frailty: Physical Activity as a Model. Cold Spring Harb Perspect Med 2016; 6:1-14.

10. Buckinx F, Rolland Y, Reginster JY, Ricour C, Petermans J, Bruyère O. Burden of frailty in elderly population: perspectives for a public health challenge. Arch Public Health 2015; 73(19):1-7.

11. Mendes EV. As redes de atenção à saúde. 2a ed. Brasília: OPAS; 2011.

12. Cirino S, Gonçalves LA, Gonçalves MB, Cursi ES, Coelho AS. Avaliação de acessibilidade geográfica em sistemas de saúde hierarquizados usando o modelo de p-medianas: aplicação em Santa Catarina, Brasil. Cad Saude Publica 2016; 32(4):1-11.

13. Silva AA. Gráficos e mapas: representação de informação estatística. Lisboa: Lidel; 2006.

14. Neri AL, Yassuda MS, Araújo LF, Eulálio MC, Cabral BE, Siqueira MEC, Santos GA, Moura JGA. Metodologia e perfil sociodemográfico, cognitivo e de fragilidade de idosos comunitários de sete cidades brasileiras: Estudo FIBRA. Cad Saude Publica 2013; 29(4):778-792. 
15. Nunes DP, Duarte YAO, Santos JLF, Lebrão ML. Rastreamento de fragilidade em idosos por instrumento autorreferido. Rev Saude Publica 2015; 49(2):1-9.

16. Ribeiro EG, Matozinhos FP, Guimarães GL, Couto AM, Azevedo RS, Mendoza IYQ. Autopercepção de saúde e vulnerabilidade clínico-funcional de idosos de Belo Horizonte/Minas Gerais. Rev Bras Enferm 2018; 71(Supl. 2):860-867.

17. Moraes EN, Carmo JA, Moraes FL, Azevedo RS, Machado CJ, Montilla DER. Índice de Vulnerabilidade Clínico Funcional-20 (IVCF-20): reconhecimento rápido do idoso frágil. Rev Saude Publica 2016; 50(81):1-10.

18. Instituto Brasileiro de Geografia e Estatístico (IBGE). Censo Demográfico 2010 [Internet]. [acessado 2018 Abr 20]. Disponível em: http://www.ibge.gov.br/ home/estatistica/populacao/censo2010/caracteristicas_da_populacao/default_caracteristicas_da_populacao.shtm

19. Brasil. Ministério da Saúde (MS). Resolução 466, de 12 de dezembro de 2012. Diretrizes e normas regulamentadoras de pesquisas envolvendo seres humanos. Diário Oficial da União 2012; 12 dez.

20. Borim FSA, Francisco PMSB, Neri AL. Fatores sociodemográficos e de saúde associados à mortalidade em idosos residentes na comunidade. Rev Saude Publica 2017; 51(42):1-12.

21. Silva SLA, Neri AL, Ferrioli E, Lourenço RA, Dias RC. Fenótipo de fragilidade: influência de cada item na determinação da fragilidade em idosos comunitários - Rede Fibra. Ciên Saude Colet 2016; 21(11):34833492.

22. Chen X, Mao G, Leng SX. Frailty syndrome: an overview. Clin Interv Aging 2014; 9:433-441.

23. Melo BRS, Diniz MAA, Casemiro FG, Figueiredo LC, Santos-Orlandi AA, Haas VJ, Orlandi FS, Gratão ACM. Avaliação cognitiva e funcional de idosos usuários do serviço público de saúde. Esc Anna Nery 2017; 21(4):1-8.

24. Côrte B, Kimura C, Ximenes MA, Nóbrega OT. Determinantes da atenção aos idosos pela rede pública de saúde, hoje e em 2030: o caso da Região Metropolitana de São Paulo. Saude Soc 2017; 26 (3):690-701.
25. Brasil. Conselho Nacional de Secretários da Saúde (CONASS). A atenção primária e as redes de atenção à saúde [Internet]. Brasília: CONASS; 2015. [acessado 2018 Mar 21]. Disponível em: http://www.conass.org. br/biblioteca/pdf/A-Atencao-Primaria-e-as-Redes-de -Atencao-a-Saude.pdf

26. Brasil. Departamento de Atenção Básica (DAB). Histórico de Cobertura da Saúde da Família [Internet]. Brasília: DAB; 2017. [acessado 2018 Fev 06]. Disponível em: http://dab.saude.gov.br/portaldab/historico_cobertura_sf.php

27. Pagliuca LMF, Lima BS, Silva JM, Cavalcante LM, Martins MC, Araújo TL. Acesso de idosos às unidades de atenção primária à saúde. REME 2017; 21:1-5.

28. Alves DSB, Barbosa MTS, Caffarena ER, Silva AS. Caracterização do envelhecimento populacional no município do Rio de Janeiro: contribuições para políticas públicas sustentáveis. Cad Saude Colet 2016; 24(1):63-69.

29. Pereira KCR, Lacerda JT, Natal S. Avaliação da gestão municipal para as ações da atenção à saúde do idoso. Cad Saude Publica 2017; 33(4):1-16.

30. Carvalho S, Magalhães MAFM, Medronho RA. Análise da distribuição espacial de casos da dengue no município do Rio de Janeiro, 2011 e 2012. Rev Saude Publica 2017; 51(79):1-10.

Artigo apresentado em 08/06/2018

Aprovado em 11/02/2019

Versão final apresentada em 13/02/2019 